\author{
Military Technical College \\ Kobry El-Kobbah, \\ Cairo, Egypt.
}

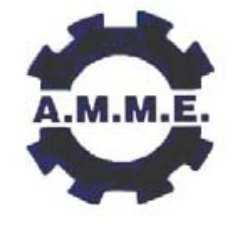

\title{
THERMAL ANALYSIS OF A MICRO DEVICE USED FOR DETECTION OF COLORECTAL CANCER
}

\author{
AHMED* M.I. and HRAIRI* M.
}

\begin{abstract}
Finite element modelling is widely used in technological applications. The benefits of using simulation are clear: reduced time and cost when introducing new products to market, better knowledge of part dynamic and static properties, and the opportunity to replace life cycle device tests, among others. Finite element analysis (FEA) is extensively used in medical devices development cycle especially during the preliminary analysis of re-design and optimization and when passing the final design analysis and reliability evaluation. Typical analysis of implantable medical devices is challenging due to the highly nonlinear nature of the physical problem due to large geometric changes, contact and material behaviour. The current paper will investigate the design of a micro device intended for detection of colorectal cancer through a thermal analysis process and optimum sizing of air slits and side cuts between the different thermal zones of the device. By comparing the results of the numerous carried out simulations, an optimum design was found.
\end{abstract}

\section{KEW WORDS}

Biomedical, Finite Element Analysis, Simulation, Medical device, Cancer

\footnotetext{
* Department of Mechanical Engineering, Faculty of Engineering, International Islamic
} University, Kuala Lumpur, Malaysia. E-mail: mirghani@iiu.edu.my. Fax: +6-03-61964455. 


\section{INTRODUCTION}

Design analysis involving the modelling of components using finite element methods enables design concepts to be evaluated and design optimisation to be achieved prior to prototype manufacture, thus saving time and cost and minimising risk. For these reasons, the power and versatility of engineering simulation has been used in developing innovative life-saving and life-enhancing biomedical products that touch the lives of millions of people around the world every day. By performing analysis up front in development, engineers have the time and resources to improve concepts, evaluate alternatives, run 'what-if' scenarios and come up with innovative designs. Biomedical simulations are many and varied, and cover all simulation disciplines, from the traditional structural analysis to fluid flow, multi-body dynamics and electromagnetics.

Some researchers used ANSYS $尺$ Structural ${ }^{\mathrm{TM}}$ software in studies to determine to what extent an artificial spinal disc helps restore motion and how performance is affected by placement relative to the centreline of the spine nucleus [1]. Others showed that FEA simulation helps design highly sensitive protein ion sources used in detecting diseases such as cancer on a molecular level at very early stages [2]. Numerical models were also used to show the stage of stent expansion, interaction of blood vessel upon the stent, what will allow determining the value of straining and stress, and the fatigue resistance of the stent [3]. Another class of device-tissue interaction involving the blood vessels has been extensively investigated by researchers to determine the material properties and constitutive models to describe the in vivo mechanical properties of blood vessels [4]. A numerical technique was also used for the design of electromagnetically coupled coils widely used by medical devices such as; cardiac pacemakers, implantable defibrillators, antitachycardia devices, drug delivery systems, artificial organs, monitoring systems as well as other stimulators use these coil systems to acquire diagnostic information and/or program the device to perform particular stimulating functions [5]. In recent years, the use of computational models for radiofrequency ablation has risen as a means of studying the dosiometric and heat transfer profiles surrounding the ablation probes. Several investigators have applied finite element analysis of hepatic radiofrequency ablation probes using temperaturedependent electrical conductivity $[6,7]$.

The objective of this paper is to show how FEA commercial packages are used to simulate the structural behaviour of medical devices at various operational stages. Specifically, how FEA is used in the design of a micro device intended for detection of colorectal cancer through a thermal analysis process and optimum sizing of air slits and side cuts between the different thermal zones of the device.

\section{MEDICAL DEVICE DEVELOPMENT CYCLE}

Before reaching the market, medical device evolution will typically include many steps starting with a conceptual idea development (material selection, manufacturability), initial drawings, design optimization, prototype development and physical testing, reliability evaluation and finally meeting packaging requirements. Figure 1 shows the American Food and Drug Administration (FDA) definition of the medical device life cycle. The stated purpose of these guidelines is to ensure the safety of the general public by preventing fraudulent or hastily developed medical products from entering the marketplace. Generally, these regulations must be applied to any software used as a 
component, part, or accessory of a medical device; any software used in the production of a device (for example, programmable automation controllers in manufacturing equipment); and any software used in the implementation of the device manufacturer's quality system (such as software that records and maintains the device history record) [8].

FEA is extensively used in this cycle especially during the preliminary analysis of redesign and optimization and when passing the final design analysis and reliability evaluation.

\section{The Medical Device}

The Ligase Detection Reaction (LDR) is a mutation detection technique used to identify point mutations in deoxyribonucleic acid (DNA). Developed by Francis Barany [9], it is used to find specific low abundant point mutations that may lead to colorectal cancer in the early stages of disease development. During LDR, several primers are mixed with the analyte, exposed to a thermal cycle. Temperatures of $0^{\circ} \mathrm{C}, 65^{\circ} \mathrm{C}$ and $95^{\circ} \mathrm{C}$ had to be obtained in order to have a successful LDR. The analyte would have to pass through $95^{\circ} \mathrm{C}$ zone for a hot start, and then the ligase enzyme, stored at $0^{\circ} \mathrm{C}$, would be added and mixed. The $95^{\circ} \mathrm{C}$ to $65^{\circ} \mathrm{C}$ temperature cycle would be repeated 20 times, at which time the reaction is stopped by cooling to $0^{\circ} \mathrm{C}$. All of the temperature regions had to be isolated and insulated from each other and the other sections of the chip, such as the mixing stages and the identification section, in order to ensure that no thermal effects occurred in those regions [10]. Hence, simulation of the thermal response of any LDR device, which should be used for detection of cancer-associated rare gene mutations, is a major step that should be done during the device design and/or operation. Figure 2 shows the layout of the LDR device that it is simulated using FEA.

\section{The Finite Element Model}

Commercial FEA package, namely Ansys, was used to simulate the behaviour of the medical device. The focus of the simulations were to look at the temperature distribution of the device layout, size the air slits, determine the power inputs needed to maintain the temperatures, and to investigate the temperature uniformity in the thermal regions.

It is known that the temperature profile within a body depends upon the rate of its internally-generated heat, its capacity to store some of this heat, and its rate of thermal conduction to its boundaries (where the heat is transferred to the surrounding environment). Mathematically, this is stated by the heat equation,

$$
\nabla^{2} T-\frac{1}{\alpha} \frac{\partial T}{\partial t}=-\frac{1}{k} \dot{q}
$$

along with its boundary conditions, equations that prescribe either the temperature $T$ on, or the heat flux $q$ through, all of the body boundaries $\Omega$,

$$
\begin{aligned}
& T\left(\Omega_{a}\right)=T_{p} \\
& q\left(\Omega_{b}\right)=q_{p} \\
& \Omega_{a} \cup \Omega_{b}=\Omega
\end{aligned}
$$


In the heat equation, the heat generated per unit volume is expressed by $\dot{q}$. The thermal diffusivity $\alpha$ is related to the thermal conductivity $k$, the specific heat $c$, and the density $\rho$ by,

$$
\alpha=\frac{k}{\rho c}
$$

For steady state and no heat generation conditions, as it is the case with the current problem, the heat equation, to be solved, simplifies to,

$$
\nabla^{2} T=0
$$

It will be assumed that all effects are in the global Cartesian system $(x, y, z)$.

Radiation, conduction and convection effects were taken into account for the simulations. Thermal loads and boundary conditions were applied. The entire chip had convective boundary condition except for the area below the thermal zone and the cooling zone. The thermal zone had a constant heat flux into to the model, while the cooling zone had a constant heat flux from the model. The line of symmetry had an adiabatic boundary condition. After loads and boundary conditions were applied the model was solved.

The structural nonlinearities inherent in medical device design are material nonlinearities (such as plasticity, super-elasticity, hyperelasticity), geometric nonlinearities (localized large strains) and contact nonlinearities.

Many simulations were carried out by studying the effect of the size of the air slits as well as by modifying their shapes. More specifically, air slits having sizes of $0.5,1,1.5$ and $2 \mathrm{~mm}$ have been simulated once without and once with side cuts. These simulations were aiming at determining the design that will provide the best temperature distribution and temperature profile. Therefore, some side cuts were introduced at the end of the slits and the same finite element simulations were run against the new geometrical models.

\section{RESULTS AND DISCUSSION}

The results showed that more than one single design will be acceptable since the temperature distribution in each thermal zone remains within its zone and did not overlap with the temperature distribution of the neighbouring zone even though, in some cases, the temperature contour propagated slightly outside the thermal cycling zone. However, the following figures show the results obtained by these FEA simulations for the retained best design of size and side cuts for the air slits. On one hand, Figure 3 shows the temperature distribution in the three thermal zones for different configurations of the device, having air slits sized $0.5,1,1.5$ and $2 \mathrm{~mm}$. On the other hand, Figure 4 displays the temperature profile in the three thermal zones for the same configuration of air slits but with the addition of side cuts. From these two figures, an optimum design is chosen. 
It can be noticed that some of the configurations show a good temperature distribution within the thermal zones without or with minimum spill outside these zones. However, when checking the temperature profile of these same configurations, a good uniformity is not achieved. Therefore, the best retained configuration should have an optimum combination of temperature distribution and profile at the same time.

More specifically, all the configurations of air slits without side cuts show a variation in the temperature profile even though the temperature distribution seems acceptable since there is no overlap of the thermal zones (Figure 3). However, with the introduction of side cuts, the temperature profiles are improving in uniformity nearing perfection for air slits sizes of 1.5 and $2 \mathrm{~mm}$. Therefore, the temperature distribution of these two configurations has to be examined in order to decide on which design should be retained as the better one. From Figure 4, it can be seen that the temperature spill outside the heating zone is more pronounced in the case of $2 \mathrm{~mm}$ air slits.

From the overall analysis of the different temperature distributions and profiles obtained for the different device configurations and presented in Figures 3 and 4, it is concluded that the LDR device having the $1.5 \mathrm{~mm}$ air slits with side cuts at their ends achieves the best temperature uniformity within the different thermal zones of the device. In addition, they are equally able to maintain good temperature isolation between the three thermal zones of the device. These two constraints on the temperature are necessary for a successful ligase detection reaction.

Figure 5 displays the temperature distribution and the temperature profiles in the different thermal regions of the device with the best design.

Referring to the medical device design life cycle (Figure 1), the current paper addresses part of the concept phase related to the preliminary analysis redesign and optimization using FEA. A logical expansion to this work would be a prototype development and its physical testing.

\section{CONCLUSION}

This paper highlights the power and versatility of engineering simulation in developing innovative life-saving and life-enhancing biomedical products that touch the lives of millions of people around the world every day. FEA is used in the design, verification, and validation of medical devices through the use of thermal and structural models. By analyzing different possible designs for an LDR device, FEA simulation results showed that a thermal profile of room temperature, $95^{\circ} \mathrm{C}$ and $0^{\circ} \mathrm{C}$ could be obtained on the device layout using air slits to separate the temperature zones. The mixing zones would be isolated, hence no thermal effects occurs in these regions. There was also no overlap between the heated and cooled thermal zones. The simulations showed good temperature uniformity inside the chambers.

\section{REFERENCES}

[1] M. Moumene and F. Geisler, "Effect of Artificial Disc Placement in Facet Loading: 
Unconstrained vs. Semi-constrained", Global Symposium on Intervertebral Disc Replacement and Non-Fusion Technology, May 2004.

[2] A. Hieke, "Ion Dynamics in Electro-Pneumatic Fields: A Key to Modern Biotechnology", ANSYS Solutions, pp. 13-17, spring 2005.

[3] J. Awrejcewicz, M. Ciach, and K. Wlodarczyk, "Finite Element Method Analysis of Non - Linear Behaviour Of Implants And Stents", ECCOMAS 2000, September 2000

[4] Y.C. Fung, "Biomechanics Mechanical Properties of Living Tissues," $2^{\text {nd }}$ Edition, Springer New York, ISBN 0-387-97947-6, 1993

[5] O.A. Mohammed and L.F. Garcia, "A Finite Element/Superposition Technique For The Design Of Electromagnetically Coupled Coils", IEEE Transactions On Magnetics, Vol. 25, No. 5, pp. 3575-3577, 1989

[6] S. Tungjitkusolmun, S.T. Staelin, D. Haemmerich, J.Z. Tsai, H. Cao, J.G. Webster, F.T. Lee, D.M. Mahvi and V.R. Vorperian, "Three-dimensional finite-element analyses for radio-frequency hepatic tumor ablation", IEEE Transactions on Biomedical Engineering, 49(1):3-9, 2002

[7] I. Chang, "Finite Element Analysis of Hepatic Radiofrequency Ablation Probesusing Temperature-Dependent Electrical Conductivity", BioMedical Engineering OnLine, 2:12, 2003 (available from http://www.biomedicalengineering-online.com/content/2/1/12)

[8] D. W. Feigal, "Total Product Life Cycle", Center for Devices and Radiological Health, FDA (www.fda.gov)

[9] F. Barany, "The ligase chain reaction (LCR) in a PCR world", PCR Methods Appl.1, pp. 5-16,1991

[10] D.O. Barrett, "Design of a Microfabricated Device for Ligase Detection Reaction (LDR)", LSU Master's Thesis, 2004. 


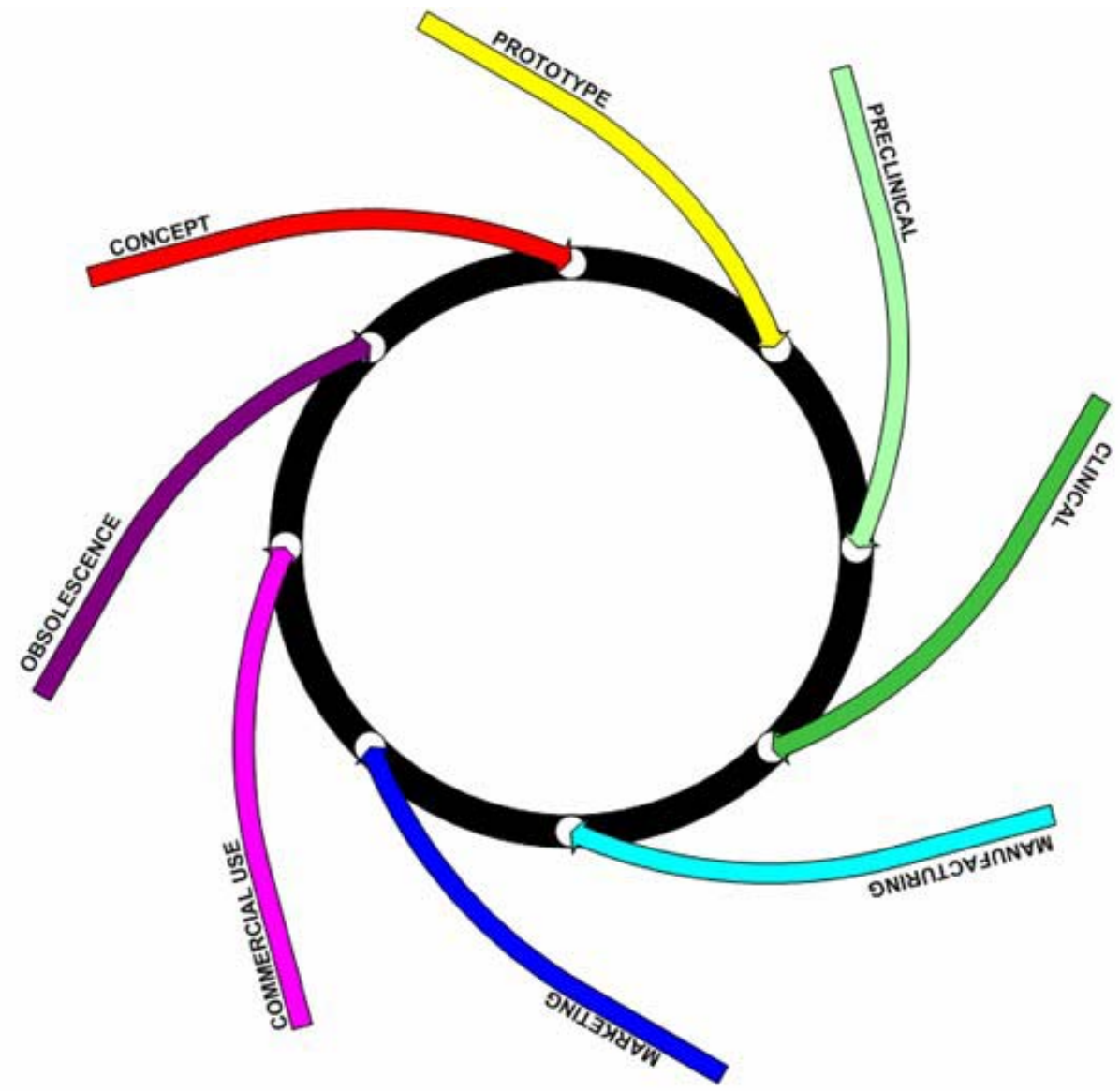

Figure 1: The medical device design life cycle as defined by the FDA [8]

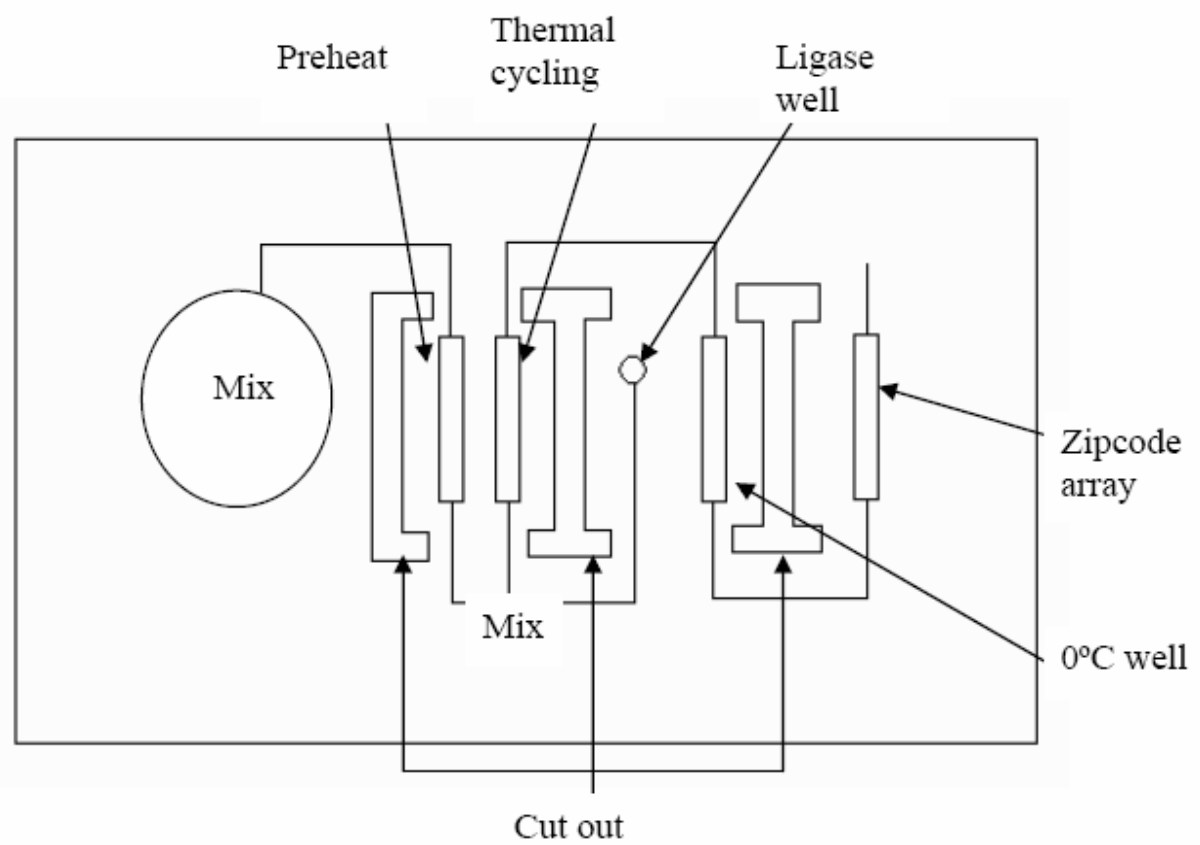

Figure 2: Layout of the LDR device [10] 

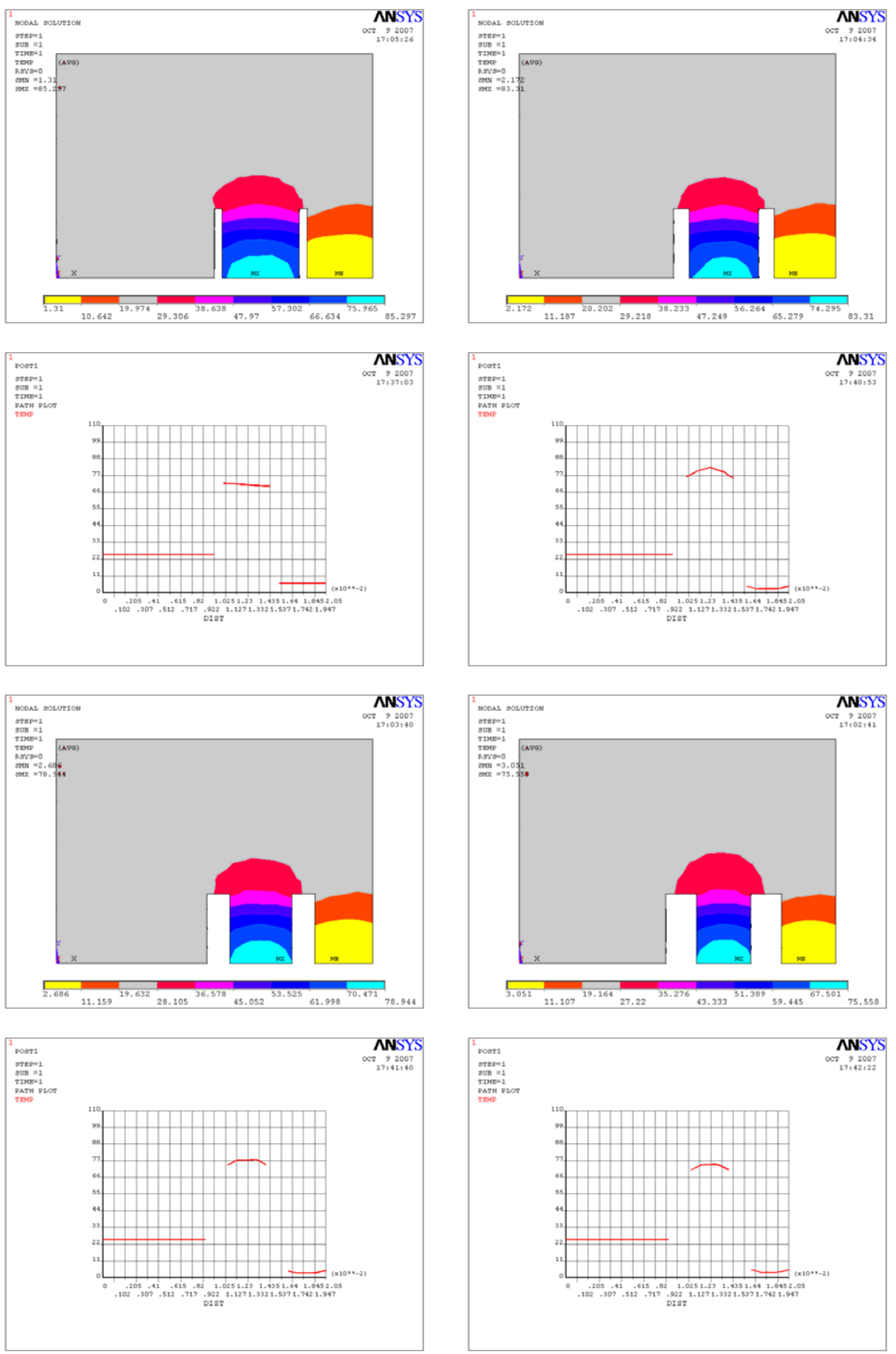

Figure 3: Temperature distribution and profile in the different thermal zones of the device for $0.5,1,1.5$ and $2 \mathrm{~mm}$ size of air slits 

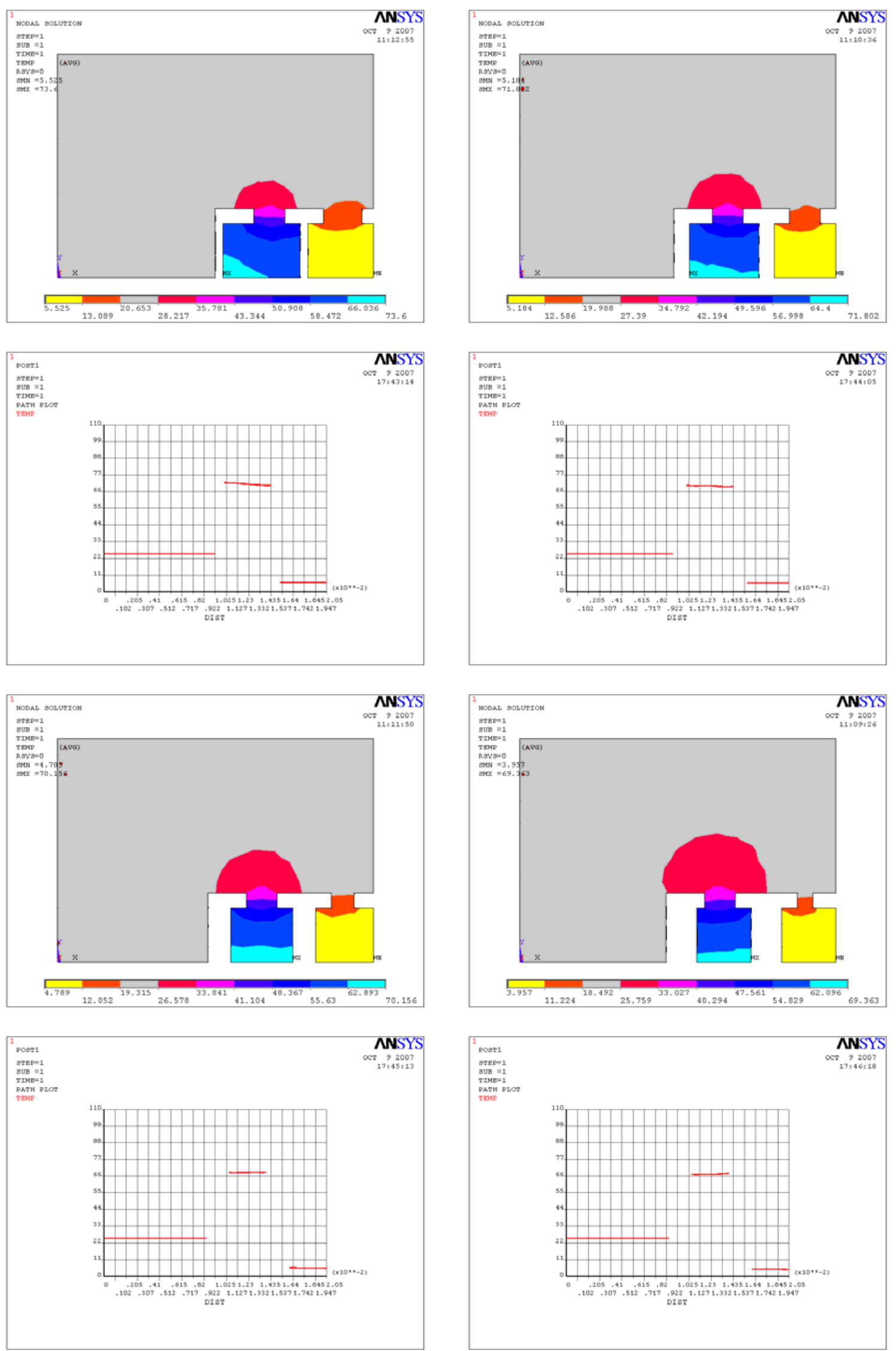

Figure 4: Temperature distribution and profile in the different thermal zones of the device for $0.5,1,1.5$ and $2 \mathrm{~mm}$ size of air slits with side cuts 

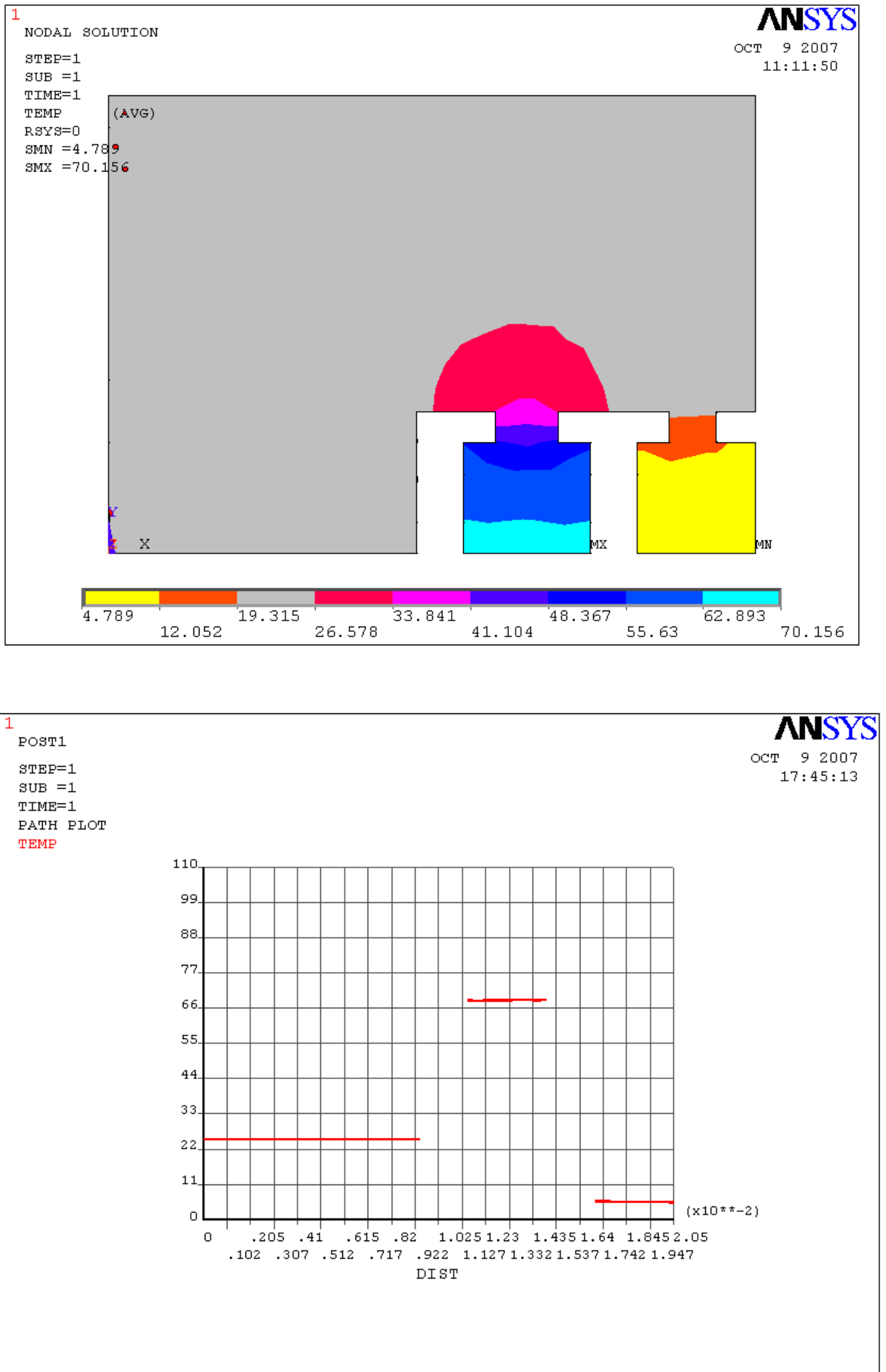

Figure 5: Temperature distribution and profile in the three thermal zones of the device having $1.5 \mathrm{~mm}$ air slits with side cuts 